\title{
Zum Arbeitsanforderungen-Arbeitsressourcen-Modell von Burnout und Arbeitsengagement - Stand der Forschung
}

\author{
Evangelia Demerouti ${ }^{1,2,3} \cdot$ Friedhelm Nachreiner $^{3}$ \\ Online publiziert: 5. Juni 2018 \\ (c) Der/die Autor(en) 2018
}

\section{Zusammenfassung}

Dieser Beitrag gibt eine Übersicht über den Stand der Forschung zum Arbeitsanforderungen-Arbeitsressourcen-Modell, welches zur Erklärung von Burnout und Arbeitsengagement dient. Dabei werden die beiden von diesem Modell angenommenen Prozesse vorgestellt und aufzeigt, wie sie mit möglichen Konsequenzen in Form von Burnout und/oder Arbeitsengagement zusammenhängen. Zusätzlich werden differentielle Zusammenhänge zwischen Arbeitsanforderungen und Arbeitsressourcen, zwischen Burnout bzw. Arbeitsengagement und weiteren Konsequenzen wie Fehlzeiten und Produktivität dargestellt. In diesem Zusammenhang werden auch Befunde von Längsschnittuntersuchungen sowie von einer Untersuchung, die auf einer objektiven Erfassung von Arbeitsbedingungen basiert, diskutiert. Darüber hinaus wird die Bedeutung individueller Ressourcen, wie das proaktive Gestalten der eigenen Arbeitstätigkeit, erläutert. Der Beitrag schließt mit praktischen Implikationen des Modells sowie den daraus abzuleitenden Empfehlungen für die Arbeits(um)gestaltung. Praktische Relevanz: Auf theoretischen Befunden basierend, demonstriert dieser Beitrag wie das ArbeitsanforderungenArbeitsressourcen-Modell zur Entwicklung und Implementierung von Arbeits(um)gestaltungsmaßnahmen genutzt werden kann. Diese Maßnahmen verfolgen das Ziel das Burnout Risiko von Beschäftigten zu reduzieren und deren Arbeitsengagement zu steigern. Interventionsstudien zeigen, dass die erfolgreiche Umsetzung der Modell-Annahmen zu positiven Ergebnisses sowohl für die Beschäftigte als auch für die Unternehmen führt.

Schlüsselwörter Burnout $\cdot$ Arbeitsengagement $\cdot$ Job Crafting $\cdot$ Arbeitsleistung $\cdot$ JD-R Modell

Evangelia Demerouti

e.demerouti@tue.nl

1 Dept. Industrial Engineering \& Innovation Sciences, Human Performance Management Group, Eindhoven University of Technology, P.O.Box 513, 5600 MB Eindhoven, Niederlande

2 Faculty of Management, University of Johannesburg, Johannesburg, Südafrika

3 GAWO e. V., Achterdiek 50, 26131 Oldenburg, Deutschland 


\title{
The job demands—job resources model of burnout and work engagement—state of the art
}

\begin{abstract}
This review provides an overview over the state of the evidence concerning the job demands-job resources model of burnout and job engagement. The two processes assumed in this model are presented, together with their potential consequences with regard to burnout and work engagement. Direct and indirect relations between job demands and job resources, burnout and engagement as well as their relations with absenteeism and productivity and the evidence available for the postulated relations are presented.

Besides results from cross sectional studies results from longitudinal studies are presented, as well as from a study using independent assessments of the independent variables. Furthermore, the importance of individual resources as well as proactive individual approaches to (re)design one's own job characteristics and activities (job crafting) are discussed on the basis of the available evidence.

The paper closes with practical implications of the JD-R model and recommendations for job (re)design.

Practical Relevance: Based on the evidence presented the review demonstrates how the JD-R model can be used to develop and implement job(re)design interventions with the goal of reducing the risk of burn out and enhancing work engagement in employees. Intervention studies show that successfully implementing the model assumptions leads to positive outcomes for employees and organizations.
\end{abstract}

Keywords Burnout $\cdot$ Work engagement $\cdot$ Job crafting $\cdot$ Job performance $\cdot$ JD-R model

\section{Einleitung}

Das Job Demands-Resources (JD-R) Modell von Demerouti und Kollegen (Demerouti et al. 2000, 2001; Bakker und Demerouti 2007, 2014, 2017; Demerouti und Bakker 2011) baut sowohl auf Job Design- als auch auf Belastungs-Beanspruchungsmodellen auf und verbindet diese. Während Job Design Theorien (z. B. Hackmann und Oldham 1980) häufig die Rolle von Anforderungen oder konkreten Belastungsbedingungen (Stressoren) übersehen, ignorieren Belastungs- oder Stresstheorien (z.B. Selye 1956) häufig das Motivationspotential arbeitsbezogener Ressourcen.

Das JD-R Modell kombiniert daher diese beiden Forschungsansätze, um zu erklären, wie Arbeitsanforderungen und -ressourcen sich sowohl eigenständig als auch in Wechselwirkung auf arbeitsbezogene Erfahrungen wie Burnout und Arbeitsengagement auswirken. Darüber hinaus prognostiziert das JD-R Modell inverse kausale Beziehungen: Während ,ausgebrannte“ Beschäftigte mit der Zeit noch mehr (wahrgenommene) Anforderungen für sich selbst generieren und berichten, mobilisieren „engagierte“ Beschäftigte ihre vorhandenen/wahrgenommenen Arbeitsressourcen, um engagiert zu bleiben.

Die bekannteste und am häufigsten verwendete Definition von Burnout stammt von Maslach und Jackson (1984); danach ist Burnout ein Syndrom, bestehend aus empfundener a) emotionaler Erschöpfung, b) Depersonalisierung und c) reduzierter persönlicher Leistungsfähigkeit, das bei Individuen, die in irgendeiner Weise mit Menschen (als Arbeitsobjekten) arbeiten, auftreten kann. Emotionale Erschöpfung bezieht sich auf das Gefühl, durch den arbeitsbezogenen Kontakt mit Menschen emotional überanstrengt und ausge- laugt zu sein. Bei der Depersonalisierung handelt es sich um das Gefühl, das abgestumpfte und zynische Reaktionen gegenüber den Empfängern der Dienstleistungen hervorruft. Reduzierte persönliche Leistungsfähigkeit beschreibt die Wahrnehmung der Abnahme eigener Kompetenz und den erlebten zunehmenden Mangel an erfolgreicher Ausführung der Arbeit mit/an Menschen.

Demerouti und Nachreiner (1998) haben mit dem Oldenburger Burnout Inventar (OLBI) ein Instrument entwickelt, das für die Erfassung von Burnout an allen, also auch an nicht (human-)dienstleistungsspezifischen Arbeitsplätzen geeignet ist, weil es (im Gegensatz etwa zum Maslach Burnout Inventory [MBI, Maslach und Jackson 1996]) keine (human-) dienstleistungsspezifischen Formulierungen einzelner Items beinhaltet. Als Dimensionen des OLBI ergeben sich daher eine empfundene a) Erschöpfung und b) Distanzierung von der eigenen Arbeit (vergleichbar der Depersonalisierung bei der Arbeit mit Menschen), welche auch als die Kerndimensionen von Burnout angesehen werden. Das Empfinden reduzierter Leistungsfähigkeit ist hierbei eher als Folge einer hohen Ausprägung der beiden Kerndimensionen zu interpretieren (Demerouti et al. 2010).

Arbeitsengagement, als weitere Folge der beiden grundlegenden Prozesse, wird in diesem Modell als positiver Antipode von Burnout gesehen. Schaufeli und Bakker (2003, 2004) definieren Arbeitsengagement als einen positiven, arbeitsbezogenen Zustand im Individuum, der charakterisiert ist durch Vitalität (vigor), Hingabe (dedication), und $\mathrm{Ab}$ sorption (vgl. auch Schaufeli et al. 2002). Arbeitsengagement bezieht sich auf einen länger andauernden affektivkognitiven Zustand, der nicht auf ein bestimmtes Objekt, Ereignis oder Individuum (als Arbeitsobjekt) beschränkt ist. 


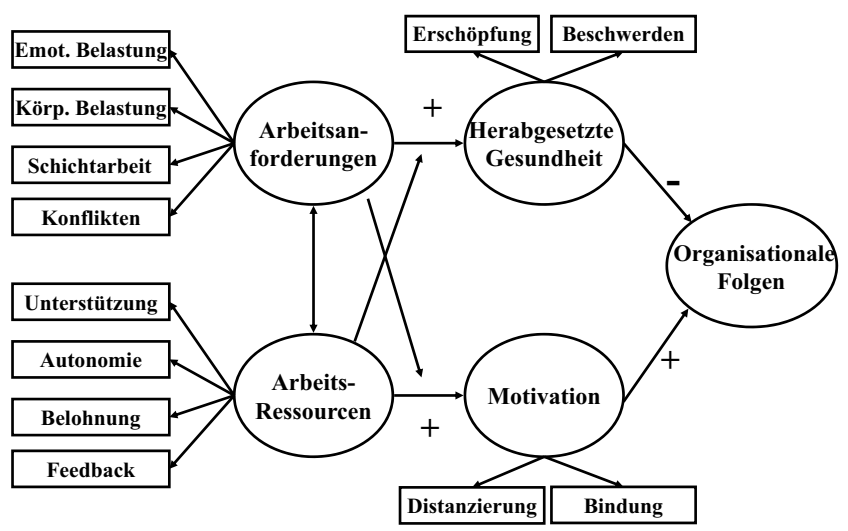

Abb. 1 Das Arbeitsanforderungen - Arbeitsressourcen Modell

Fig. 1 The job demands - job ressources model (JD-R Model)

Vitalität ist gekennzeichnet durch ein hohes Ausmaß von Energie und psychischer Spannkraft während der Arbeit und die Bereitschaft sich bei der Arbeit trotz aller Schwierigkeiten anzustrengen. Hingabe bezieht sich auf das Empfinden von Wichtigkeit, Enthusiasmus, Inspiration und Herausforderung bezüglich der eigenen Arbeit. Vitalität ist der Gegenpol zu Erschöpfung, während Hingabe das Gegenteil von Depersonalisierung, Zynismus (Schaufeli und Bakker 2003) oder Distanzierung von der Arbeit (Demerouti und Nachreiner 1998) ist. Die dritte Dimension, Absorption, hat sich aus 30 Tiefeninterviews als ein Bestandteil von Arbeitsengagement ergeben (Schaufeli und Bakker 2003). Absorption ist gekennzeichnet durch volle Konzentration sowie das Gefühl, durch die Arbeit gefesselt zu sein, und dass dabei die Zeit verfliegt.

Dieser Beitrag gibt eine Übersicht über den derzeitigen (Beginn 2018) Stand der Forschung zum JD-R (oder auf Deutsch Arbeitsanforderungen - Arbeitsressourcen) Modell (Demerouti et al. 2000, 2001) und seine Weiterentwicklung. Weil das Modell von großer Relevanz sowohl für die Forschung als auch für die betriebliche Praxis ist, hat dieser Beitrag das Ziel statt einer ausführliche Literaturrecherche oder etwa einer Meta-Analyse eine narrative Übersicht über den derzeitigen Stand der Dinge zu geben. Für weitere Überblickspublikationen über das Modell wie auch MetaAnalysen verweisen wir auf Bakker und Demerouti (2014, 2017) sowie auf Nahrgang et al. (2011). Auf diese Weise wollen wir die Anwendung und Diskussion des Modells, dessen internationale Originalveröffentlichung (Demerouti et al. 2001) bis Ende 2017 mehr als 2650-mal zitiert wurde, auch im deutschsprachige Raum, in dem es übrigens entstanden ist, weiter stimulieren.

Das JD-R Modell wird eingesetzt, um Burnout und dessen positiven Antipoden „Arbeitsengagement“ vorherzusagen (vgl. Abb. 1). Dabei nimmt das Modell an, dass Burnout und Arbeitsengagement die Folgen zweier unterschiedlicher Prozesse sind: eines Beeinträchtigungsprozesses und eines Motivierunsprozesses, die beide durch die Arbeitsaufgaben und ihre Ausführungsbedingungen ausgelöst werden. Wir fokussieren uns hier auf Burnout und Arbeitsengagement, weil sich die Forschung zu diesem Modell insbesondere auf die Entwicklung von Burnout und Arbeitsengagement konzentriert, auch wenn im Laufe der Zeit Untersuchungen dazu gekommen sind, die auch andere Konsequenzen des Modells untersucht haben. Dabei wird auf die empirischen Befunde zu diesem Modell aus Quer- und Längsschnittuntersuchungen eingegangen, wie auf eine Untersuchung, die auf objektiven Erhebungen von Arbeitsbedingungen basiert.

\section{Das „Arbeitsanforderungen- Arbeitsressourcen-Modell“}

Die Vielfalt der verschiedenen arbeitsbezogenen Ursachen von Burnout wurde von Demerouti (1999) und Demerouti et al. $(2000,2001)$ in einem Modell zusammengefasst, das allgemeine Belastungs- und Motivationstheorien integriert und von zwei Grundannahmen ausgeht.

Die erste Grundannahme ist, dass die Diversität von unterschiedlicher Risikofaktoren für das Entstehen von Burnout in zwei breiten Kategorien bzw. Konstrukten zusammengefasst/vereinfacht werden kann: Arbeitsanforderungen und Arbeitsressourcen. Arbeitsanforderungen repräsentieren physische, psychische, soziale und organisatorische Aspekte der Arbeit, die eine, in der Regel länger andauernde, physische und/oder psychische Anspannung erfordern, und demzufolge mit bestimmten physiologischen und/oder psychischen Kosten zusammenhängen. Beispiele solcher Arbeitsanforderungen sind hohe zeitliche und inhaltliche Anforderungen (erlebbar als „Arbeitsdruck“), eine aversive Arbeitsumgebung (z.B. chemische Stoffe oder Lärm) oder emotionale Anforderungen durch enge zeitliche Bindung oder anspruchsvolle Kunden, die z.B. durch Beschäftigte in Dienstleistungsberufen in der Regel als herausfordernd empfunden werden (Bakker und SanzVergel 2013).

Arbeitsressourcen sind die physischen, psychischen, sozialen und organisatorischen Arbeitsbedingungen, die (1) funktional für das Erreichen der arbeitsbezogenen Ziele sind, (2) Arbeitsanforderungen und damit zusammenhängende physische und psychische Kosten reduzieren und (3) persönliches Wachstum und persönliche Entwicklung stimulieren. Arbeitsressourcen beziehen sich auf die Organisation (z. B. Partizipation an Entscheidungen, Arbeitsplatzsicherheit, Belohnungen, Aufstiegsmöglichkeiten), die Aufgaben, ihre Ausführungsbedingungen und Folgen (z. B. Rückmeldung über die Leistung durch Kunden und Vorgesetzte, Vielfalt von Aufgaben, wie etwa therapeutische und administrative Aufgaben, Selbstbestimmungsmöglichkei- 
ten), oder auch auf die zwischenmenschlichen Beziehungen (z.B. soziale Unterstützung durch Kollegen und Führungskräfte, Kooperations- und Kommunikationsmöglichkeiten).

Die zweite Annahme des Modells ist, dass die Entwicklung von Burnout oder Arbeitsengagement eine Folge von zwei unterschiedlichen Prozessen ist (vgl. Abb. 1). In dem ersten Prozess führt die Konfrontation mit hohen oder schlecht gestalteten Arbeitsanforderungen längerfristig zur Entstehung von Erschöpfung. Ein solcher Prozess wird schon in den fünfziger Jahren von Selye (1956) in seinem Modell zur „Allgemeinen Adaptation“ angesprochen. Nach Selye führt ein lang andauerndes Ausgesetztsein gegenüber einem Stressor (oder einer Arbeitsanforderung im JD$\mathrm{R}$ Modell) und ein wiederholt erfolgloser Versuch diesen Stressor zu bewältigen, zum Aufbrauchen der körperlichen Energiereserven und darüber zur physischen Erschöpfung. Auch wenn Arbeitsanforderungen nicht notwendigerweise per se negativ sind bzw. negative Effekte bewirken, können sie zu Stressoren mit negativen Konsequenzen werden, wenn die Bewältigung der Anforderung (insbesondere auf Dauer) hohen Einsatz erfordert, von dem der Beschäftigte sich nicht angemessen erholen kann (Meijman und Mulder 1998). Es handelt sich hier also um einen Prozess der Konsumption (individueller, physischer wie psychischer) Ressourcen.

Im zweiten Prozess wird wegen eines Mangels an hinreichenden Arbeitsressourcen das Erreichen der Arbeitsziele erschwert oder behindert, und demzufolge entstehen Gefühle der Frustration und des Scheiterns. Diese Gefühle vermindern die Motivation, weitere (oder auch dieselben) zukünftige Arbeitsziele anzustreben oder zu erreichen. Die Konsequenz davon ist die Entstehung einer emotional negativ besetzten Distanzierung von und eines Zynismus gegenüber der eigenen Arbeit, sowie in der Folge das Gefühl der herabgesetzten Leistungsfähigkeit; wohingegen im positiven Fall der Bewältigung der Arbeitsaufgabe und der Zielerreichung mit Hilfe der vorhandenen Ressourcen Arbeitsengagement entsteht. Die Arbeitsressourcen können daher im Sinne von motivationalen Anreizen verstanden werden, wie sie auch im Job Characteristics Modell (JCM) von Hackman und Oldham (1980) angesprochen werden. Nach Hackman und Oldham (1980) erhöhen Arbeitsbedingungen bzw. -merkmale wie Aufgabenvariabilität, -signifikanz, -identität sowie Autonomie und Rückmeldung das motivationale Potential an einem Arbeitsplatz und stehen daher mit erhöhter Motivation und gesteigertem Engagement in Verbindung.

Zusätzlich zeigen die vorliegenden Ergebnisse (Demerouti et al. 2000, 2001), dass der Einfluss der Arbeitsanforderungen auf die Distanzierung von der Arbeit und das Arbeitsengagement relativ unbedeutend ist. Gleiches gilt für den Einfluss der Arbeitsressourcen auf die Erschöpfung. So ist zum Beispiel bei einem Arbeitsplatz eines Elektrotechnikers mit hoher Arbeitsintensität und unregelmäßigen
Arbeitszeiten, schlechten Umgebungsbedingungen, und hohen kognitiven Anforderungen bei der Lösung von technischen Problemen zu erwarten, dass mit der Zeit Erschöpfungsgefühle, nicht aber Distanzierung von der eigenen Arbeit entstehen werden. Andererseits ist, wenn man Anerkennung durch Vorgesetzte für die eigene Leistung erfährt, oder Selbstbestimmungsmöglichkeiten über die Arbeitsweise und/oder Entwicklungsmöglichkeiten (z. B. über Trainingsmaßnahmen) hat, die Entstehung von Arbeitsengagement zu erwarten, während beim Fehlen dieser Ressourcen Distanzierung von der Arbeit erwartet werden sollte.

\section{Empirische Befunde über die Prädiktoren von Burnout und Arbeitsengagement}

Die Gültigkeit der durch das Modell angenommenen $\mathrm{Zu}$ sammenhänge wurde nicht nur unter Verwendung subjektiv eingeschätzter Arbeitsbedingungen (also Anforderungen und Ressourcen) durch die Betroffenen, sondern auch mit Hilfe objektiver, von der Zielperson unabhängiger, bedingungsbezogener Messungen der Anforderungen und Ressourcen als Merkmalen der Arbeitssituation (und nicht des Individuums) bestätigt. So wurden in der Studie von Demerouti (1999) die Arbeitsanforderungen und -ressourcen an verschiedenen Arbeitsplätzen mit Hilfe eines Arbeitsanalyseverfahrens von einem externen Beobachter eingeschätzt. Das Verfahren wurde für die Zwecke dieser Studie entwickelt und basierte auf vorhandenen Instrumenten (zum Beispiel dem Instrument zur Stressbezogenen Tätigkeitsanalyse; Semmer et al. 1999). Zusätzlich wurden die Mitarbeiter nach denselben Arbeitsbedingungen sowie nach ihrem Burnout befragt (personenbezogene Erfassung). Die Ergebnisse bestätigen, dass die subjektiv erlebten Arbeitsanforderungen und -ressourcen das individuelle, personenbezogene Burnout (mit dem Beschäftigten als Merkmalsträger) vorhersagen konnten, während die objektiv erfassten Arbeitsanforderungen und -ressourcen das arbeitsplatzbezogene Burnout (als Mittelwert über alle Mitarbeiter eines Arbeitsplatzes, und damit dem Arbeitsplatz als Merkmalsträger) vorhersagen konnten. Die Arbeitsanforderungen waren verantwortlich für die Erfahrung von Erschöpfung, die Arbeitsressourcen hingegen für die Distanzierung von der Arbeit, unabhängig davon, ob sie objektiv oder subjektiv erfasst wurden, oder Burnout als individuelles oder arbeitsplatzbezogenes Merkmal konzipiert wurde.

Das bedeutet, dass die durch das Modell angenommenen Prozesse nicht nur Antworttendenzen oder interne Muster von subjektiven Repräsentationen der Arbeitssituation darstellen, sondern auch objektiv, also unabhängig von den zu ihrem Burnout-Erleben befragten Personen, feststellbar und veränderbar sind. Darüber hinaus weisen die Ergebnisse darauf hin, dass Burnout nicht nur als individuelles, per- 
sonenbezogenes Konstrukt verstanden werden muss, sondern ebenfalls als ein dem Arbeitsplatz und seinen konkreten Arbeitsbedingungen als Merkmalsträger zuordenbares Konstrukt aufgefasst werden kann. Diese Erkenntnis lässt schlussfolgern, dass zur Prävention von Burnout bedingungsbezogene gegenüber individuellen, personenbezogenen Präventionsansätze zu präferieren sind.

Die Grundannahmen des Modells sind durch Untersuchungen an verschiedenen Populationen, Arbeitsplätzen und in verschiedenen Ländern bestätigt worden (vgl. die Übersicht in Bakker und Demerouti 2007, 2014, 2017). Interessant ist dabei, dass, obwohl die zwei angenommenen Prozesse an unterschiedlichen Arbeitsplätzen grundsätzlich bestätigt werden, sich allerdings die spezifischen, jeweils konkret gegebenen Indikatoren der Arbeitsanforderungen und -ressourcen für die jeweiligen Funktionen/ Arbeitskonstellationen unterscheiden können. Dies ist aber keine Einschränkung, sondern spricht vielmehr für die Validität und die Verallgemeinerbarkeit des Modells und der darin verwendeten Konstrukte.

Die vorliegenden Studien über die Antezedenzien von Burnout und Arbeitsengagement zeigen konsistent, dass die beiden (Ziel-)Zustände durch unterschiedliche Prädiktoren vorhergesagt werden können. Demerouti et al. (2000, 2001) haben deutlich gezeigt, dass die zwei zentralen Komponenten von Burnout jeweils durch unterschiedliche konkrete Bedingungen prognostiziert werden. Während Erschöpfung das Resultat von hohen oder schlecht gestalteten Arbeitsanforderungen (unterschiedlichster Art) ist, wird die Distanzierung von der Arbeit jeweils durch fehlende Arbeitsressourcen bedingt. Mit anderen Worten ist Burnout eine Folge von hohen Arbeitsanforderungen und fehlenden Arbeitsressourcen, während fehlendes Arbeitsengagement dominant eine Folge von fehlenden Arbeitsressourcen zu sein scheint.

Diese Beziehungen sind nicht nur bei Querschnittstudien, sondern auch bei Langschnittstudien nachgewiesen worden. Demerouti et al. (2009) haben mit einer dreistufigen Längschnittstudie gezeigt, dass Krankenschwestern, die mit subjektiv anspruchsvollen Patienten, einer hohen Arbeitsbelastung und erheblichen körperlichen Anforderungen konfrontiert waren, ein Jahr später mehr Erschöpfung und eine stärker negativ ausgeprägte Einstellung gegenüber ihren Patienten (d.h. Depersonalisation) entwickelten als bei der Vormessung. Dies ist ein alarmierender Befund, da er auf eine (rückgekoppelte) Verschlechterung der Pflegequalität über die Zeit hinweg hinweisen könnte. Darüber hinaus bietet die Studie von Demerouti et al. (2009) Belege für die Hypothese der inversen Kausalität und deutet damit auf eine ,Verlust Spirale“ bei Burnout hin. Beide Komponenten von Burnout, emotionale Erschöpfung und Depersonalisierung, hatten zu den späteren Erhebungszeitpunkten positive Auswirkungen auf die subjektiv wahrgenommenen und genannten Arbeitsanforderungen. Eine daraus sich ergebende Frage ist natürlich, ob und wie Burnoutgefühle die objektiven, von der Person unabhängigen Arbeitsanforderungen verändern können, oder ob es sich hier lediglich um die veränderte Wahrnehmung dieser Bedingungen handelt.

Vergleichbare Resultate über den Motivationsprozess haben Xanthopoulou et al. (2009) gefunden. Diese Längsschnittstudie untersuchte zyklische Beziehungen zwischen Arbeitsressourcen, persönlichen Ressourcen und Arbeitsengagement. Die Studie wurde an 163 Mitarbeitern durchgeführt, die über einen Zeitraum von 2 Jahren verfolgt wurden. Es wurde festgestellt, dass Arbeits- und persönliche Ressourcen zum Zeitpunkt 1 positiv mit dem Arbeitsengagement zum Zeitpunkt 2 verbunden waren. Zusätzlich hing Arbeitsengagement zum Zeitpunkt 1 positiv mit Arbeitsund persönlichen Ressourcen zum Zeitpunkt 2 zusammen. Das Modell, das die Daten am besten wiederspiegelt, war ein reziprokes Modell, welches davon ausging, dass nicht nur Ressourcen und Arbeitsengagement, sondern auch die wahrgenommenen Arbeits- und persönlichen Ressourcen miteinander verbunden waren. Diese Ergebnisse unterstützen die Annahme, dass sich verschiedene Arten von Ressourcen und Wohlbefinden in einem positiven Rückkopplungsprozess entwickeln, welcher die erfolgreiche Anpassung von Arbeitnehmern an ihre Arbeitsumgebungen durch motivationale Prozesse mitbestimmt.

\section{Zu den Konsequenzen von Burnout und Arbeitsengagement}

Nachfolgende Untersuchungen haben das Modell erweitert, einerseits durch die Berücksichtigung anderer Indikatoren von Gesundheit und Motivation als Erschöpfung und Distanzierung von der Arbeit und andererseits durch die Untersuchung verschiedener Konsequenzen von Burnout und Arbeitsengagement. In der Untersuchung von Bakker et al. (2003a) ist Bindung an die Organisation als ein Indikator der Motivation mit aufgenommen worden. Bindung an die Organisation wurde dabei operationalisiert als affektive Bindung (d.h. die positive emotionale Bindung des Mitarbeiters an seine Organisation), normative Bindung (d.h. die Vor- und Nachteile des Arbeitens in einer Organisation) sowie Kontinuitätsbindung (d.h. das Verbleiben in einer Organisation auf der Basis empfundener Verpflichtungen) (Meyer und Allen 1991). Die Ergebnisse weisen darauf hin, dass Arbeitsanforderungen durch ihre Wirkung auf das Burnout einen Effekt auf die Dauer der durch die Organisation registrierten individuellen Fehlzeiten (welche als Indikator der Gesundheit betrachtet werden können) der Mitarbeiter hatten. Arbeitsressourcen hingegen wirkten sich auf die Bindung an die Organisation aus, was wiederum einen Effekt auf die Häufigkeit der Fehlzeiten hatte (Arbeitsressourcen 
können somit als ein möglicher Indikator der Motivation betrachtet werden; cf. ,withdrawal“ hypothesis, Johns 1997). Auch wenn damit keine kausale oder zeitbezogene Verbindung nachgewiesen werden kann, weisen die Ergebnisse auf die zu erwartende Assoziation von motivationalen wie auch gesundheitlichen Indikatoren mit objektiv registriertem tatsächlichem Mitarbeiterverhalten hin.

In einer Untersuchung von 146 Mitarbeitern aus unterschiedlichen Berufen wurde das Arbeitsanforderungen-Arbeitsressourcen Modell genutzt um die durch Kollegen eingeschätzten Intra- und Extra-Rollen Leistung (Bakker et al. 2004) vorherzusagen. Intra-Rollen Leistung wird definiert als die offiziell erforderlichen Ergebnisse und Verhaltensweisen, die die Erreichung der Organisationsziele direkt ermöglichen (Motowidlo und Van Scotter 1994), wie z. B. das Erfüllen der Produktivitätsziele und das effektive Funktionieren der Organisation (Behrman und Perreault 1984). Extra-Rollen Leistung bezieht sich auf freiwilliges Verhalten von Mitarbeitern, das keinen direkten Einfluss auf die Produktivität der Person hat, aber das effektive Funktionieren der Organisation fördert (Podsakoff und MacKenzie 1994). Die Intra-Rollen Leistung wies dabei einen starken negativen Zusammenhang mit Erschöpfung und einen schwächeren, negativen Zusammenhang mit Arbeitsanforderungen auf, während die Extra-Rollen Leistung ausschließlich durch Distanzierung von der Arbeit (ebenfalls ein negativer Zusammenhang) vorhergesagt wurde.

\section{Wechselwirkungen zwischen Arbeitsanforderungen und Ressourcen}

Neben den angenommenen und inzwischen belegten Haupteffekten der Arbeitsanforderungen und der Arbeitsressourcen schlägt das JD-R Modell vor (Bakker und Demerouti 2007, 2014, 2017), dass auch Wechselwirkungen zwischen Arbeitsanforderungen und Arbeitsressourcen von Bedeutung für die Entstehung arbeitsbezogener Beanspruchungen und ihrer Folgen sowie auch der Motivation sind.

Inhärenter Bestandteil der Konzeption der Arbeitsressourcen ist die Annahme, dass Arbeitsressourcen den Einfluss der Arbeitsanforderungen auf die Arbeitsbeanspruchung abpuffern oder moderieren können (s. oben), einschließlich der Entstehung von Burnout (Bakker et al. 2005, 2003b). Diese Annahme einer Pufferfunktion der Arbeitsressourcen ist konsistent mit den Annahmen im Demand-Control Modell (DCM; Karasek 1998) und im Effort-Reward Imbalance Modell (ERIM; Siegrist 1996). Während das DCM aussagt, dass die wahrgenommene Kontrolle über die Ausführung von Aufgaben (Autonomie) den Einfluss der Überlastung auf die resultierende Stessreaktion puffert und das ERIM davon ausgeht, dass Belohnungen die aversiven Effekte der Ausübung von An- strengung minimieren, erweitert das JD-R Modell diese Sichtweisen und postuliert, dass die veschiendenen Arten von Arbeitsanforderungen und Arbeitsressourcen bei der Vorhersage von Arbeitsbeanspruchung (und damit bei den unterstellten konsumptiven Prozessen) interagieren können. Welche der Arbeitsanforderungen und -ressourcen in einer bestimmten Organisation und bei der Erfüllung bestimmter Arbeitstätigkeiten eine Rolle spielen, hängt dabei von den jeweils vorherrschenden Merkmalen der Tätigkeit ab, ist also, wie nach der Definition von Anforderungen und Ressourcen als übergreifende Konstrukte mit jeweils spezifischen Indikatoren (s. oben) zu erwarten, jobspezifisch.

Eine neuere Vorhersage des JD-R Modells ist, dass Arbeitsressourcen die Motivation und das Arbeitsengagement insbesondere dann beeinflussen, wenn die Arbeitsanforderungen hoch sind. Dies bezieht sich auf die sogenannte Coping-Hypothese (Bakker et al. 2007, 2010). So fanden Bakker et al. (2007), dass Arbeitsressourcen (Innovativität, Wertschätzung und ein positives Organisationsklima) besonders vorteilhaft bei der Aufrechterhaltung von Arbeitsengagement waren, in Situationen mit hohen Arbeitsanforderungen (z.B. Fehlverhalten von Schülern). Vergleichbar dazu fanden Bakker et al. (2010), dass Arbeitsressourcen (Nutzung von Fertigkeiten, Lernmöglichkeiten, Autonomie, Unterstützung durch Kollegen und Vorgesetzte, Leistungsrückmeldung, Teilnahme an Entscheidungen und Karrieremöglichkeiten) die Freude bei der Aufgabenerfüllung und die Verbundenheit mit der Organisation insbesondere unter Bedingungen hoher Arbeitsanforderungen (Leistungssdruck und emotionale Anforderungen) prognostizierten. Auch das weist darauf hin, dass Ressourcen insbesondere in Situationen mit hohen Anforderungen eine erhöhte Bedeutung zukommt. Dies ist nachvollziehbar, wenn man bedenkt, dass für die Bewältigung hoch anfordernder Arbeitsaufgaben und -bedingunen eben ausreichende Ressourcen erforderlich sind.

\section{Die Rolle des Individuums}

Als eher arbeitspsychologisches, bedingungsorientiertes Modell hat das JD-R Modell anfänglich die Rolle der Person ,,ignoriert“, weil diese im Kontext der Gestaltung der Arbeitsbedingungen von geringerer Relevanz ist (vgl. Gestaltung der Arbeitsbedingungen für definierte Zielgruppen (z.B. 5. bis 95. Perzentil), und nicht für Individuen, insbesondere im Kontext eines kollektiv organisierten Arbeitsschutzes oder mehrfach besetzter Schichtarbeitsplätze). Im Laufe der Zeit wurde das Individuum jedoch auf zwei Wegen in das Modell integriert. Zunächst besteht eine wichtige Erweiterung des JD-R Modells in der Aufnahme personaler Ressourcen in das Modell. Xanthopoulou et al. (2007) überprüften die Rolle dreier personaler Ressourcen 
(Selbstwirksamkeit, organisationsbasierte Selbstachtung und Optimismus) in der Vorhersage von Erschöpfung und Arbeitsengagement. Personale Ressourcen wurden dabei z.T. als moderierende Faktoren der Beziehung zwischen Arbeitsressourcen und Arbeitsengagement erkannt, was darauf hinweist, dass Arbeitsressourcen die Entwicklung personaler Ressourcen fördern (vgl. auch Kohn und Schooler 1982, zu intellektuellen Ressourcen). Auch eine Längsschnittstudie von Xanthopoulou et al. (2009) legt nahe, dass personale Ressourcen sich im Zeitverlauf reziprok zu Arbeitsressourcen und Arbeitsengagement entwickeln. Auf diese Weise sagen die berichteten Arbeitsressourcen die späteren personalen Ressourcen sowie Arbeitsengagement voraus; und personale Ressourcen und Arbeitsengagement wiederum sagen die später berichteten Arbeitsressourcen voraus. Eine daraus sich ergebende Frage ist natürlich, ob es sich hier lediglich um die Reduzierung kognitiver Dissonanzen zwischen der Wahrnehmung der Arbeitsressourcen, der personalen Ressourcen und des eigenen Zustands handelt.

Zweitens wurde das Individuum als aktiver Agent in das Modell integriert, das die eigenen (Arbeits-)Anforderungen und Ressourcen (um)gestaltet. Das Modell behauptet, dass das Vorhandensein von gut gestalteten und erträglichen Arbeitsanforderungen sowie von angemessenen Arbeitsressourcen negative Beanspruchungsfolgen reduzieren und die Motivation fördern kann. Was geschieht aber, wenn solche günstigen Bedingungen nicht gegeben sind? Beschäftigte könnten, sofern möglich, aktiv die Ausgestaltung ihrer Tätigkeit ändern, indem sie andere Aufgaben wählen, über unterschiedliche Arbeitsinhalte verhandeln und ihren Aufgaben oder Tätigkeiten Bedeutsamkeit zuschreiben, die bisher nicht erkannt/beachtet wurden (Parker und Ohly 2008). Dieser Prozess, wonach die Beschäftigten ihre Tätigkeit formen, wird als ,job crafting“ (Anpassung der Tätigkeit) bezeichnet (Wrzesniewski und Dutton 2001). Job crafting wird dabei definiert als die physischen und kognitiven Veränderungen, die Individuen an ihren Aufgaben oder relationalen Grenzen vollziehen. Physische Änderungen beziehen sich auf die Form, den Umfang oder die Anzahl von Arbeitsaufgaben, während kognitive Veränderungen sich darauf beziehen, wie die Tätigkeit wahrgenommen und bewertet wird. Wrzesniewski und Dutton (2001) weisen darauf hin, dass Job Crafting per se für eine Organisation weder gut noch schlecht ist. Seine Auswirkungen hängen vielmehr von der Situation und der Richtung der Veränderungen ab. Job Crafting repräsentiert den aktiven Gebrauch von Handlungs- und Tätigkeitsspielräumen, um die eigene Arbeit (den eigenen Ansprüchen nach) anzupassen und nicht lediglich die Verfügbarkeit von Handlungs- und Tätigkeitsspielräumen (dies wären in dem JD-R Modell Arbeitsressourcen und Merkmale der Situation). Demzufolge ist Job Crafting eine Strategie, die Individuen gebrau- chen (können), und damit ein individuelles Merkmal anstatt eines Arbeitsplatzmerkmals. Ein Arbeitsplatzmerkmal ist die Möglichkeit, in seinem Job Job Crafting einzusetzen, und damit eine Arbeitsressource. Job Crafting kann aber zu Veränderungen in den Arbeitsmerkmalen führen, wenn Menschen erfolgreich im Ausführen und Umsetzen der erwünschten Veränderungen sind.

In jüngster Zeit wird Job Crafting auch über die Veränderungen definiert, die Beschäftigte bezüglich ihrer Arbeitsanforderungen und Arbeitsressourcen umsetzen können (Petrou et al. 2012; Tims et al. 2012). Diese Konzeptualisierung nutzt das JD-R Modell als Ausgangssituation. Job Crafting kann dabei verschiedene Formen des Verhaltens annehmen: (a) Vergrößerung der (strukturellen und sozialen) Arbeitsressourcen; (b) Vergrößerung (d.h. anspruchsvollere) Arbeitsanforderungen; (c) Verringerung (hinderlicher) Arbeitsanforderungen (Petrou et al. 2012; Tims et al. 2012). Tims et al. (2012) fanden, dass SelbstBerichte über Job Crafting positiv korrelierten mit der Fremdeinschätzung durch Kollegen bezüglich des Arbeitsengagements, der Beschäftigungsfähigkeit und der Leistung und dass Selbsteinschätzungen des Job Craftings ebenfalls positiv mit Fremdeinschätzungen durch Gleichgestellte korrelierten. Das weist darauf hin, dass Job Crafting Verhaltensweisen repräsentiert, die auch von anderen wahrgenommen werden können.

Darüber hinaus zeigten Tims et al. (2013), dass Beschäftigte, die ihre Arbeitsressourcen in den ersten Monaten der Untersuchung (um)gestalteten, eine Steigerung ihrer (selbst berichteten) strukturellen und sozialen Ressourcen im Verlauf der Studie (zwei Monate) erzielten. Diese (wahrgenommene) Vergrößerung der Arbeitsressourcen war verbunden mit verstärktem Arbeitsengagement und größerer Arbeitszufriedenheit. Vergleichbar dazu fanden Petrou et al. (2012) in ihren Tagebuchstudien, dass an Tagen mit empfundenem hohem Leistungsdruck (hohe Arbeitsanforderungen) und gleichzeitig hoher Autonomie (hohe Arbeitsressourcen, d.h. aktive Tätigkeiten nach dem Karasek-Modell) die Beschäftigten ihre Ressourcen stärker vergrößerten und ihre Anforderungen weniger reduzierten. Interessanterweise konnte gezeigt werden, dass je mehr die Beschäftigten an einem bestimmten Tag anstrebten ihre Arbeitsanforderungen zu erhöhen, umso mehr waren sie in ihrer Tätigkeit engagiert. Im Gegensatz dazu erlebten Beschäftigte umso weniger Engagement an einem Tag, je mehr sie versucht hatten, ihre Tätigkeit zu vereinfachen. Von daher scheinen Job Crafting oder eine bottom-up Anpassung der Anforderungen und Ressourcen eine bedeutsame Rolle in den vom JD-R Modell vorgeschlagenen Mechanismen/ Zusammenhängen zu spielen. 


\section{Auf dem JD-R Modell basierende Empfehlungen für die Arbeits(um)gestaltung}

JD-R Studien haben übereinstimmend gezeigt, dass die Beschäftigten in der Regel ihre besten Leistungen in herausfordernden, ressourcenreichen Arbeitsumgebungen zeigen, weil solche Arbeitsumgebungen ihr Arbeitsengagement fördern (Bakker und Bal 2010; Demerouti und Cropanzano 2010). Dies impliziert, dass Organisationen ihren Mitarbeitern hinreichende Arbeitsressourcen bieten sollten - einschließlich Rückmeldung, sozialer Unterstützung, einer Vielfalt anzuwendender Fertigkeiten - sowie eine Optimierung der Arbeitsanforderungen in einer Form, die diese erfüllbar macht. Dies kann sowohl durch top-down Ansätze geschehen, wie organisationale Human Ressources Management Aktivitäten und Führungstrainings, wie auch durch bottom-up Ansätze, einschließlich Job Crafting durch die Mitarbeiter.

Bezüglich organisationaler Ansätze fanden Boon und Kalshoven (2014), dass HR-Praktiken mit hohem Einsatz, wie etwa kontinuierliches Training, hohe Arbeitsplatzsicherheit, Übertragung von Verantwortlichkeiten, Job Rotationen, einem Fokus auf Lernen und entwicklungsbezogenem Feedback, variable Entlohnung und ein umfangreiches Vorteils-Paket positiv mit Arbeitsengagement und organisationalem Commitment verbunden waren. Dies gilt vor allen Dingen, wenn das Niveau der Aufgabenerfüllung ressourcenbedingt gering war, d.h. unter Bedingungen, wo den Beschäftigten die Ressourcen fehlten, die sie brauchten, um ihre Aufgaben zu bewältigen. Das deutet darauf hin, dass HR Praktiken dazu beitragen können, fehlende Ressourcen zu kompensieren oder zu überwinden.

Andererseits fanden Jensen et al. (2013), dass Hochleistungs-Arbeitssysteme, die auf eine Steigerung der Wettbewerbsfähigkeit der Organisation ausgelegt sind, dies auf Kosten der Beschäftigten realisieren. Dabei fanden sie, dass Hochleistungs-Arbeitssysteme insbesondere zu Rollenüberlastung und Ängsten der Beschäftigten beitrugen, wenn die Möglichkeiten zur Gestaltung der Arbeitsbedingungen gering waren. Diese Ergebnisse weisen deutlich darauf hin, dass die Implementierung organisationaler Praktiken mit einer Steigerung der Ressourcen der einzelnen Beschäftigten verbunden sein sollte, anderenfalls können sie unliebsame Effekte auf die Arbeitsanforderungen und darüber auf das Wohlbefinden der Beschäftigten ausüben.

Im Hinblick auf die Rolle von Führungskräften zeigen Forschungsergebnisse, dass Vorgesetzte die Arbeitsanforderungen und Arbeitsressourcen ihrer Mitarbeiter beeinflussen können (Nielsen et al. 2008), und darüber indirekt das Engagement der Mitarbeiter und deren Leistung (Tims et al. 2011). So sind etwa Fernet et al. (2015) in ihrer Studie bei Pflegekräften und Schulleitern dem Einfluss der Führung auf die Leistung der Geführten nachgegangen. In Übereinstimmung mit dem JD-R Modell fanden sie, dass transformative Führung (d.h. Führungskräfte, die in der Lage sind, ihre Mitarbeiter durch inspirierende Motivation, individuelle Rücksichtnahme und intellektuelle Stimulierung $\mathrm{zu}$ inspirieren, Aufwand in gemeinsame Ziele zu investieren) zu geringeren Arbeitsanforderungen (kognitive, emotionale und physische Anforderungen) führte und indirekt eine positivere Einstellung zur Arbeit und bessere Arbeitsleistung der Mitarbeiter hervorrief. Das JD-R Modell bietet damit im Kontext des Trainings von Führungskräften einen nützlichen Rahmen für die Spezifikation der günstigsten Konstellation der Arbeitsbedingungen.

Im Kontrast zu traditionellen Ansätzen der Umgestaltung von Arbeitstätigkeiten im Kontext von top-down Ansätzen, stellt Job Crafting eine Intervention auf individuellem Niveau dar, die typischerweise von den einzelnen Beschäftigten initiiert wird, und zwar in Form eines bottom-up Ansatzes. Organisationen können Job Crafting Verhaltensweisen, die für Mitarbeiter wie für Organisationen gleichermaßen vorteilhaft sind, anregen, indem sie den Mitarbeitern zeigen, wie sie ihre Tätigkeiten erfolgreich gestalten können.

Van den Heuvel et al. (2015) haben eine solche Intervention anhand von Polizisten entwickelt und überprüft. Mit Hilfe verschiedener Erklärungen und Übungen im Rahmen von Workshops haben die Beschäftigten das Konzept des Job Craftings kennengelernt und wurden instruiert, ihren eigenen Personal Crafting Plan (PCP) zu entwickeln. Dieser PCP bestand aus verschiedenen Aktivitäten zum Job Crafting, die die Teilnehmer durchzuführen hatten. Während eines Zeitraums von vier aufeinanderfolgenden Wochen vergrößerten die Teilnehmer ihre (wahrgenommenen) herausfordernden Anforderungen und verringerten ihre (wahrgenommenen) hinderlichen Arbeitsanforderungen. Die Teilnehmer tauschten auch ihre Crafting Erfahrungen während eines Reflektions-Treffens aus, wobei sie Erfolge, Probleme und Lösungen diskutierten. Es zeigte sich, dass die Intervention die Wahrnehmung/Beurteilung zweier Arbeitsressourcen vergrößerte (Kontakt mit dem Vorgesetzten sowie Möglichkeiten der eigenen Weiterentwicklung), eine personale Ressource (Selbstwirksamkeit) sowie das Wohlbefinden verbesserte, wobei die Teilnehmer über mehr positive und weniger negative Emotionen berichteten. In einer weiteren Interventionstudie bei Mitarbeitern eines Arbeitsamtes haben Hulshof et al. (2016) gezeigt, dass Kunden von Mitarbeitern die an der Job Crafting Intervention teilgenommen haben, zufriedener waren als Kunden von Mitarbeitern der Kontrollgruppe. Das impliziert, dass die Job Crafting Intervention zu Verbesserungen geführt hat, die durch unabhängige Personen beobachtet und berichtet werden konnten.

Ein entscheidender Unterschied zwischen top-down und bottom-up Ansätzen besteht dabei darin, dass top-down 
Ansätze dieselben Arbeitsmerkmale für alle Beschäftigten vergrößern (obwohl nicht alle Mitarbeiter diese Merkmale gleichermaßen wertschätzen), während eine bottom-up Crafting Intervention darin resultiert, dass jeder Mitabeiter unterschiedliche Merkmale versucht zu verändern, und zwar je nach den eigenen Präferenzen (Demerouti 2014).

Die spannende Frage ist, ob, oder bis zu welchem Grad mitarbeiterinitiierte Job Crafting Ansätze die top-down Ansätze ersetzen können. Bottom-up Ansätze sind zu begrüßen, weil sie dazu beitragen können, die Passung zwischen Job und individuellen Präferenzen zu verbessern, auch wenn wir davon ausgehen, dass die Organisation für das Wohlbefinden der Mitarbeiter und die Erreichung der Organisationsziele verantwortlich ist. Um dies zu erreichen, brauchen Organisationen und Führungskräfte neue Formen des Managements, bei dem die Führungskräfte ihren Mitarbeitern die Möglichkeit bieten, ihre Jobs zu gestalten, gleichzeitig aber Grenzen aufzeigen, indem sie die Verhaltensweisen kommunizieren und praktizieren, die im jeweiligen organisationalen Kontext sowohl für die Mitarbeiter wie auch für die Organisation zulässig (z. B. im Kontext der Arbeits- und Systemsicherheit), sinnvoll und günstig sind.

\section{Schwächen des Modells}

Neben den hier vorgestellten Stärken des JD-R Modells sollen dessen bisher noch bestehende, aber zukünftig durch entsprechende Anlagen der Studien zu behebende, insbesondere methodologische, Schwächen nicht verschwiegen werden. Wie auch die oben angeführten ähnlichen Modelle (DCM, ERIM, JCM s. oben), die positive und negative Reaktionen der Beschäftigten mit positiven und negativen Tätigkeitskomponenten zu erklären versuchen, unterstellt das JD-R Modell Zusammenhänge zwischen den konkreten Arbeitsbedingungen und den individuellen, subjektiven Reaktionen der Betroffenen. Dabei erfolgt die Messung/ Erfassung der Arbeitsbedingungen (der hypothetisch positiv sowie negativ wirkenden auf positive wie auch negative Konsequenzen) über eine Messung der von den Betroffenen subjektiv erfahrenen, widergespiegelten und bewerteten Arbeitsbedingungen, ohne dass deren bedingungsbezogene Messgüte (vgl. DIN EN ISO 10075-3:2004; Nachreiner 2012) in Bezug auf Objektivität, Reliabilität, Validität, Sensitivität und Diagnostizität hinreichend abgeklärt wäre. Dies aber ist für eine bedingungsbezogene Interpretation der Forschungsergebnisse, wie sie in der Regel erfolgt, unerlässlich.

Im Gegensatz zu den anderen Vertretern der Modellfamilie, die dieses Problem zu ignorieren scheinen und personenorientierte Messungen ohne jeden Nachweis der Zulässigkeit dieses Vorgehens bedingungsorientiert interpretieren, nähert sich lediglich Demerouti (1999), wenn auch nur in einer einzigen Untersuchung, dieser Problematik empirisch an. In dieser Untersuchung analysierte Demerouti die Übereinstimmung der per Fragebogen von den Betroffenen erhobenen Daten zu den Arbeitsbedingungen mit den per unabhängigen, nicht betroffenen Beobachtern dazu erhobenen Daten. Die Ergebnisse dieser Überprüfung zeigen, dass die Übereinstimmung zwischen beiden Datenquellen ausgesprochen defizitär ist. So sind die Korrelationen mit wenigen Ausnahmen und in der Regel<0,7, für einige Dimensionen sind sie sogar (signifikant) negativ. Damit kann nicht davon ausgegangen werden, dass beide Zugänge dasselbe intendierte Merkmal - die tatsächliche Beschaffenheit der tatsächlichen Arbeitsbedingungen - erfassen. Offensichtlich, und wie unter Berücksichtigung der Tatsache, dass es sich um Daten auf der Basis sozialer Wahrnehmung handelt, enthalten demnach die im Kontext des JD-R Modells von den Betroffenen erhobenen subjektiven Daten zur Charakterisierung der Arbeitsbedingungen erhebliche subjektive (Bewertungs-) Varianzanteile. Sie enthalten damit unter der Perspektive der intendierten Erfassung der tatsächlichen Arbeitsbedingungen erhebliche validitätsbeeinträchtigende Stör- oder Fehlervarianzanteile. Insofern ist deren Verwendung und Interpretation als bedingungsbezogene Messung mit erheblichen Vorbehalten zu begegnen. Da bisher nur Demerouti (1999) Arbeitsanforderungen und -Ressourcen mit einem objektiven Verfahren gemessen hat, sind weitere Studien zu diesem Problem (auch bei den anderen Modellen dieser Modellfamilie) dringend erforderlich, um abklären zu können, inwieweit die Datenerhebungen der unabhängigen Variablen per Fragebogen über die Ergebnisse sozialer Wahrnehmungs- und Urteilsbildungsprozess die postulierten Variablen tatsächlich und valide erfassen; oder ob es sich dabei um durch die im Modell als abhängig betrachteten Variablen beeinflusste Variablen, und damit tatsächlich um abhängige Variablen handelt. Wichtig und dringlich ist damit (und zwar innerhalb der gesamten Modellfamilie) eine Abklärung der bedingungsbezogenen Validität der Erfassung der Arbeitsanforderungen (und nicht der wahrgenommenen oder subjektiv beurteilten Arbeitsanforderungen) und der Arbeitsressourcen (und nicht der empfundenen Arbeitsressourcen). Als Methode der Wahl bietet sich dazu eine generalisierbarkeitstheoretische Vorgehensweise an (vgl. Cronbach et al. 1972), und zwar in Übereinstimmung mit den Vorgaben der DIN EN ISO 10075-3:2004 (vgl. Nachreiner 2002, 2008, 2012) zur Messung der psychischen Belastung. Sollten sich dabei keine hinreichenden Anteile der Zielvarianz (d.h. der auf die Arbeitsbedingungen entfallenden Varianz, unabhängig vom Beurteiler) ergeben, müsste das Modell auf Aussagen über Zusammenhänge zwischen subjektiv perzipierten Inhalten beschränkt und der Anspruch auf Erklärung von Zusammenhängen zwischen tatsächlichen Arbeitsbedingungen und den Reaktionen der Beschäftigten aufgegeben werden. 
Wenn jedoch nur die Erklärung von Zusammenhängen zwischen subjektiv perzipierten/beurteilten Bedingungen und subjektiven Reaktionen beansprucht wird, sollte allerdings geprüft werden, inwieweit die berichteten Zusammenhänge auch unter und jenseits der Berücksichtigung der nach Festinger (1957) zu erwartenden Konsistenzen aussagekräftig sind. Die Konstruktion des Modells (und insbesondere auch der vergleichbaren Modelle aus der Modellfamilie), wonach positiv bewertete Arbeitsbedingungen mit positiv bewerteten Ergebnisvariablen zusammenhängen (und vice versa), dürfte derartige konsistente Bewertung geradezu herausfordern, was die berichteten Zusammenhänge, zumindest teilweise, ebenfalls erklären könnte.

Insofern muss das Modell dringend gegen diese konkurrierenden Hypothesen abgesichert werden. Unserer Auffassung nach am besten durch die Nutzung unterschiedlicher und nicht einer potentiellen Dissonanzreduktion unterliegenden Datenquellen und deren jeweilige theoretisch angemessene, also bedingungsbezogene, Validierung, wenn die tatsächlichen Bedingungen den Merkmalsträger und den Erfassungsgegenstand darstellen. Dagegen sind personenbezogene Validierungen erforderlich, wenn die Merkmalsträger, über die Aussagen intendiert sind, die einzelnen Beschäftigten und deren subjektive Beurteilungen ihrer Arbeitsbedingungen sind.

Diese Überlegungen gelten nicht nur für Querschnittsuntersuchungen. Auch für Längsschnittuntersuchungen oder für Interventionsstudien muss abgesichert werden, wem die erhobenen Messwerte als Merkmalsträger (Personen oder Arbeitsbedingungen, und in welchem Umfang) zuzuordnen sind. Anderenfalls werden sich auch dabei Probleme mit und Bedrohungen der Validität der behaupteten Zusammenhänge ergeben. Für ein eher arbeitspsychologisches, bedingungsorientiertes Modell wie das JD-R Modell erscheinen solche Überlegungen zentral, insbesondere weil etwa aus dem Kontext des JCM bekannt ist, dass sich die Relationen und deren Stärke drastisch verändern können, wenn wirklich einmal ausnahmsweise Daten aus unabhängigen Quellen verwendet werden (vgl. Algera 1990). Der weiteren Forschung zum JD-R Modell muss daher daran gelegen sein, diese zentrale Validitätsbedrohung des Modells zu entkräften - auch um sich von den anderen Modellen der Modellfamilie deutlich abzusetzen.

Obwohl das Modell in verschiedenen Studien und Meta-Analysen bestätigt wurde und damit auf die Validität der unterstellten Zusammenhänge (allerdings ohne Berücksichtigung der oben vorgestellten Messprobleme) hinweist (Crawford et al. 2010; Halbesleben 2010; Nahrgang et al. 2011) besteht ein Problem des Modells darin, dass seine Bestätigung auf seiner Flexibilität beruhen kann, die erlaubt dass alle Formen von Anforderungen, Ressourcen und Ergebnissen bei seiner Überprüfung einbezogen werden können. Diese Flexibilität des Modells macht es in vielen ver- schiedenen Kontexten anwendbar, allerdings auf Kosten einer eingeschränkten Generalisierbarkeit (Schaufeli und Taris 2014). Wird zum Beispiel eine Beziehung zwischen Arbeitsdruck und Erschöpfung gefunden, dann bedeutet das nicht, dass ähnliche Relationen zwischen allen Anforderungen und allen gesundheitsbezogenen Ergebnisvariablen bestehen. Darüber hinaus haben Schaufeli und Taris (2014) andere kritische Kommentare bezüglich des Modells aufgeworfen und auf ungelöste Probleme hingewiesen, u.a.: (1) den erkenntnistheoretischen Status des Modells, weil es auf anderen Theorien aufbaut, um die beiden Prozesse zu erklären, (2) die Natur von und konzeptuelle Unterschiede zwischen Anforderungen und Ressourcen, (3) die unklare Rolle persönlicher Ressourcen, (4) die weniger präzise Unterscheidung von Gesundheits- und Motivationsprozess, (5) die bessere Integration reziproker Verursachung zwischen Anforderungen und Ressourcen auf der einen Seite und zwischen den Ergebnisvariablen auf der anderen Seite, (6) die Integration mehrstufiger Konzepte (z. B. die Rolle von Team oder organisationalen Konstrukten).

\section{Schlussfolgerungen}

Das vorgestellte Arbeitsanforderungen-Arbeitsressourcen Modell kann als ein heuristisches Modell genutzt werden, um für neu oder umzugestaltende Arbeitsplätze ungünstig gestaltete Arbeitsanforderungen und unzureichende Arbeitsressourcen zu identifizieren und umzugestalten, so dass als deren Folge Erschöpfung und Distanzierung von der Arbeit minimiert und gleichzeitig das Arbeitsengagement erhöht werden können. Obwohl das ArbeitsanforderungenArbeitsressourcen Modell (Demerouti et al. 2000, 2001) sich in die Tradition des allgemeineren Demand-Control Modells (DCM; Karasek 1998) wie des Effort-RewardImbalance-Modells (ERIM; Siegrist 1996) einfügt, erfüllt es auch das Erfordernis der Spezifität der Arbeitsbedingungen, einschließlich verschiedener Arten von Arbeitsanforderungen und Ressourcen, und zwar abhängig von dem jeweiligen beruflichen und betrieblichen Kontext der Untersuchung. Insgesamt ergeben die empirischen Befunde unterstützende Belege für die Idee, dass Arbeitsanforderungen und Ressourcen für zwei verschiedene Prozesse verantwortlich sind. Demnach sind Arbeitsanforderungen (wie im Modell konzipiert) verantwortlich für die Entstehung von Erschöpfung und gesundheitlichen Beeinträchtigungen, während Arbeitsressourcen (ebenfalls wie im Modell konzipiert) mit Motivation und Engagement zusammenhängen. Weil Burnout sowohl durch Erschöpfung als auch durch Distanzierung von der Arbeit als konstitutionelle Bestandteile gekennzeichnet ist, ist es modelltheoretisch (und wie die bisher vorliegenden empirischen Ergebnisse nahelegen) ein Resultat beider Prozesse. Querschnitts- wie 
auch Längsschnittsuntersuchungen lassen vermuten, dass Beeinträchtigungen der Gesundheit und der Motivation am Arbeitsplatz sowohl Ergebnisse als auch Prädiktoren von Arbeitsanforderungen und Ressourcen sein können, so dass herabgesetzte Gesundheit und beeinträchtigte Motivation im Laufe der Zeit zu weniger günstigen Arbeitsbedingungen (oder deren Beurteilung) führen. Damit deutet sich hier ein Rückkopplungsprozess an, der mit einer Gefahr des Aufschaukelns und schließlich des Zusammenbruchs des Systems droht. Dabei bezieht sich dieser Rückkopplungsprozess in diesem Kontext auf die betroffenen Individuen, sofern keine Intervention erfolgt.

Abschließend ist zu bemerken, dass das JD-R Model erfolgreich erweitert wurde durch die Aufnahme der Rolle personaler Ressourcen in den Motivationsprozess wie auch der Rolle des Individuums als aktiv handelndes Subjekt, das die es sich umgebenden Arbeitsanforderungen und -ressourcen (aus)gestalten kann. Auf diese Weise kann das JD-R Modell genutzt werden, um sowohl top-down wie bottom-up Ansätze der Umgestaltung von Arbeitstätigkeiten anzuleiten, um gesunde, motivierte und optimal operierende Beschäftigte zu erhalten/bewahren. Zukünftige Untersuchungen sollten nicht nur die Anwendung des JD$\mathrm{R}$ Modells bei unterschiedlichen Arbeitsplätzen/Berufen untersuchen, sondern auch häufiger objektive, personenunabhängige und bedingungsorientiert überprüfte Messungen der Arbeitsanforderungen und der Arbeitsressourcen einsetzen. Auch für die Erfassung der postulierten Konsequenzen ließen sich z.T. andere Operationalisierungen (z.B. physiologische Messungen, beobachtbares Verhalten) einsetzen. Mit dem Ziel der Entwicklung, Umsetzung und Validierung erfolgversprechender und erfolgreicher Arbeits(um)gestaltungsmaßnahmen, würde somit dem Vorwurf des Methodenmonismus entgegengetreten und eine angemessene Validitätsüberprüfung des Modells vorgenommen werden können.

Open Access Dieser Artikel wird unter der Creative Commons Namensnennung 4.0 International Lizenz (http://creativecommons.org/ licenses/by/4.0/deed.de) veröffentlicht, welche die Nutzung, Vervielfältigung, Bearbeitung, Verbreitung und Wiedergabe in jeglichem Medium und Format erlaubt, sofern Sie den/die ursprünglichen Autor(en) und die Quelle ordnungsgemäß nennen, einen Link zur Creative Commons Lizenz beifügen und angeben, ob Änderungen vorgenommen wurden.

\section{Literatur}

Algera JA (1990) The job characteristics model of work motivation revisited. In: Kleinbeck U, Quast H-H, Thierry H, Häcker H (Hrsg) Work motivation. Lawrence Erlbaum, Hillsdale

Bakker AB, Bal PM (2010) Weekly work engagement and performance: a study among starting teachers. J Occup Organ Psychol 83:189-206

Bakker AB, Demerouti E (2007) The job demands-resources model: state of the art. J Manage Psychol 22:309-328
Bakker AB, Demerouti E (2014) Job demands - resources theory. In: Chen PY, Coopers CL (Hrsg) Work and wellbeing. Wellbeing: a complete reference guide, Bd. III. Wiley-Blackwell, Chichester, S $37-64$

Bakker AB, Demerouti E (2017) Job demands-resources theory: taking stock and looking forward. J Occup Health Psychol 22:273-285

Bakker AB, Sanz-Vergel AI (2013) Weekly work engagement and flourishing: the role of hindrance and challenge demands. J Vocat Behav 83:397-409

Bakker AB, Demerouti E, De Boer E, Schaufeli WB (2003a) Job demands and job resources as predictors of absence duration and frequency. J Vocat Behav 62:341-356

Bakker AB, Demerouti E, Taris TW, Schaufeli WB, Schreurs PJG (2003b) A multi-group analysis of the job demands - resources model in four home-care organizations. Int J Stress Manag $10: 16-38$

Bakker AB, Demerouti E, Verbeke W (2004) Using the job demands resources model to predict burnout and performance. Hum Resour Manage 43:83-104

Bakker AB, Demerouti E, Euwema MC (2005) Job resources buffer the impact of job demands on burnout. J Occup Health Psychol 10:170-180

Bakker AB, Hakanen JJ, Demerouti E, Xanthopoulou D (2007) Job resources boost work engagement, particularly when job demands are high. J Educ Psychol 99:274-284

Bakker AB, Van Veldhoven MJPM, Xanthopoulou D (2010) Beyond the demand-control model: thriving on high job demands and resources. J Pers Psychol 9:3-16

Behrman DN, Perreault WDJ (1984) A role stress model of the performance and satisfaction of industrial salespersons. J Mark 48:9-21

Boon C, Kalshoven K (2014) How high-commitment HRM relates to engagement and commitment: the moderating role of task proficiency. Hum Resour Manage 53:403-420

Crawford ER, LePine JA, Rich BL (2010) Linking job demands and resources to employee engagement and burnout: a theoretical extension and meta-analytic test. J Appl Psychol 95:834-848

Cronbach LJ, Gleser GC, Nanda H, Rajaratnam N (1972) The dependability of behavioral measurements. Wiley, New York

Demerouti E (1999) Burnout: eine Folge konkreter Arbeitsbedingungen bei Dienstleistungs- und Produktionstatigkeiten. Kontrolltätigkeiten. Peter Lang, Frankfurt/Main

Demerouti E (2014) Design your own job through job crafting. Eur Psychol 19:237-247

Demerouti E, Bakker AB (2011) The job demands-resources model: challenges for future research. SA J Ind Psychol 37(2):Art. \#974

Demerouti E, Cropanzano R (2010) From thought to action: employee work engagement and job performance. In: Bakker AB, Leiter MP (Hrsg) Work engagement: a handbook of essential theory and research. Psychology Press, New York, S 147-163

Demerouti E, Nachreiner F (1998) Zur Spezifität von Burnout für Dienstleistungsberufe: Fakt oder Artefakt. Z Arbeitswiss 52:82-89

Demerouti E, Bakker AB, Nachreiner F, Schaufeli WB (2000) A model of burnout and life satisfaction among nurses. J Adv Nurs 32:454-464

Demerouti E, Bakker AB, Nachreiner F, Schaufeli WB (2001) The job demands-resources model of burnout. J Appl Psychol 86:499-512

Demerouti E, Le Blanc P, Bakker AB, Schaufeli WB (2009) Absent or present: a three-wave study on presenteeism. Career Dev Int 14:50-68

Demerouti E, Mosterd K, Bakker AB (2010) Burnout and work engagement: a thorough investigation of the Independency of both constructs. J Occup Health Psychol 15:209-222

DIN EN ISO 10075-3:2004 Ergonomische Grundlagen bezüglich psychischer Arbeitsbelastung - Teil 3: Grundsätze und Anforderungen an Verfahren zur Messung und Erfassung psychischer Arbeitsbelastung. Beuth, Berlin

Fernet C, Trépanier S, Austin S, Gagné M, Forest J (2015) Transformational leadership and optimal functioning at work: on the me- 
diating role of employees' perceived job characteristics and motivation. Work Stress 29:11-31

Festinger L (1957) A theory of cognitive dissonance. Row Peterson, Evanston

Hackman JR, Oldham GR (1980) Work redesign. Addison-Wesley, Reading

Halbesleben JRB (2010) A meta-analysis of work engagement: Relationships with burnout, demands, resources, and consequences. In: Leiter MP, Bakker AB (Hrsg) Work engagement: a handbook of essential theory and research. Psychology Press, New York, S $102-117$

Hulshof, I.L., Demerouti, E. \& Le Blanc, P.M. (2016) Providing services in times of change: A job crafting intervention to enhance performance and to protect employee well-being. Unpublished manuscript, Eindhoven University of Technology, The Netherlands

Jensen JM, Patel PC, Messersmith JG (2013) High-performance work systems and job control consequences for anxiety, role overload, and turnover intentions. J Manage 39:1699-1724

Johns G (1997) Contemporary research on absence from work: correlates, causes and consequences. Int Rev Ind Organ Psychol 12:115-173

Karasek RA (1998) Demand/Control Model: A social, emotional, and physiological approach to stress risk and active behaviour development. In: Stellman JM (Hrsg) Encyclopaedia of occupational health and safety. ILO, Geneva, S 34.6-34.14

Kohn ML, Schooler C (1982) Job conditions and personality: a longitudinal assessment of their reciprocal effects. Am J Sociol 87:1257-1286

Maslach C, Jackson SE (1984) Burnout in organizational settings. In: Oscamp S (Hrsg) Applied social psychology annual, Bd. 5, S $133-153$

Maslach C, Jackson SE (1996) Maslach burnout inventory-human services survey (MBI-HSS). In: Maslach C, Jackson SE, Leiter MP (Hrsg) MBI manual, 3. Aufl. Consulting Psychologists Press, Palo Alto

Meijman TF, Mulder G (1998) Psychological aspects of workload. In: Drenth PJ, Thierry H, de Wolff CJ (Hrsg) Handbook of work and organisational psychology, 2. Aufl. Erlbaum, Hove, S 5-33

Meyer JP, Allen NJ (1991) A three-component conceptualization of organizational commitment. Hum Resour Manage Rev 1:61-89

Motowidlo SJ, Van Scotter JR (1994) Evidence that task performance should be distinguished from contextual performance. J Appl Psychol 79:475-480

Nachreiner F (2002) Über einige aktuelle Probleme der Erfassung, Messung und Beurteilung der psychischen Belastung und Beanspruchung. Z Arbeitswiss 56:10-21

Nachreiner F (2008) Erfassung psychischer Belastung und Rückwirkung auf die Arbeitsgestaltung. Grenzen der Aussagekraft subjektiver Belastungsanalysen mithilfe von Befragungsinstrumenten aus arbeitswissenschaftlicher Sicht. Leistung und Lohn. Z Arbeitswirtsch 445-449:7-28

Nachreiner F (2012) Entwicklung und aktuelle Bedeutung der Normenreihe DIN EN ISO 10075. In: Demerouti E, Fergen A, Glaser J, Herbig B, Hofmann A, Nachreiner F, Packebusch L, Sailer K, DIN e. V. (Hrsg) Psychische Belastung und Beanspruchung am
Arbeitsplatz. Inklusive DIN EN ISO 10075-1 bis -3. Beuth, Berlin, Wien, Zürich, S 8-16

Nahrgang JD, Morgeson FP, Hofmann DA (2011) Safety at work: a meta-analytic investigation of the link between job demands, job resources, burnout, engagement, and safety outcomes. J Appl Psychol 96:71-94

Nielsen K, Randall R, Yarker J, Brenner S (2008) The effects of transformational leadership on followers' perceived work characteristics and psychological well-being: a longitudinal study. Work Stress 22:16-32

Parker SK, Ohly S (2008) Designing motivating jobs. In: Kanfer R, Chen G, Pritchard R (Hrsg) The organizational frontiers series: Vol. 27. Work motivation: Past, present, and future. Routledge, New York, S 233-284

Petrou P, Demerouti E, Peeters M, Schaufeli WB, Hetland J (2012) Crafting a job on a daily basis: contextual antecedents and the effect of work engagement. J Organ Behav 33:1020-1104

Podsakoff PM, MacKenzie SB (1994) Organizational citizenship behaviors and sales unit effectiveness. J Mark Res 31:351-363

Schaufeli WB, Bakker AB (2003) Burnout en bevlogenheid. In: Schaufeli WB, Bakker AB, de Jonge J (Hrsg) De psychologie van arbeid en gezondheid. Bohn Stafleu Van Loghum, Houten, S 295-310

Schaufeli WB, Bakker AB (2004) Job demands, job resources, and their relationship with burnout and engagement: a multi-sample study. J Organ Behav 25:293-315

Schaufeli WB, Taris TW (2014) A critical review of the Job DemandsResources Model: Implications for improving work and health. In: Bauer G, Hämmig O (Hrsg) Bridging occupational, organizational and public health. Springer, Dordrecht, S 43-68

Schaufeli WB, Salanova M, Gonzalez-Roma V, Bakker AB (2002) The measurement of engagement and burnout: a confirmative analytic approach. J Happiness Stud 3:71-92

Selye H (1956) Annual report of stress. McGraw-Hill, New York

Semmer N, Zapf D, Dunckel H (1999) Instrument zur stressbezogenen Tätigkeitsanalyse (ISTA). In: Dunckel H (Hrsg) Handbuch psychologischer Arbeitsanalyseverfahren. Mensch, Technik, Organisation, Bd. 14. vdf, Zürich, S 179-204

Siegrist J (1996) Adverse health effects of high effort-low reward conditions. J Occup Health Psychol 1:27-41

Tims M, Bakker AB, Xanthopoulou D (2011) Do transformational leaders enhance their followers' daily work engagement? Leadersh Q 22(1):121-131

Tims M, Bakker AB, Derks D (2012) The development and validation of the Job Crafting Scale. J Vocat Behav 80:173-186

Tims M, Bakker AB, Derks D (2013) The impact of job crafting on job demands, job resources, and well-being. J Occup Health Psychol 18:230-240

Van den Heuvel M, Demerouti E, Peeters MCW (2015) The job crafting intervention: effects on job resources, self-efficacy, and affective well-being. J Occup Organ Psychol 88:511-532

Wrzesniewski A, Dutton JE (2001) Crafting a job: revisioning employees as active crafters of their work. Acad Manage Rev 26:179-201

Xanthopoulou D, Bakker AB, Demerouti E, Schaufeli WB (2007) The role of personal resources in the job demands-resources model. Int J Stress Manag 14:121-141

Xanthopoulou D, Bakker AB, Demerouti E, Schaufeli WB (2009) Reciprocal relationships between job resources, personal resources, and work engagement. J Vocat Behav 74:235-244 